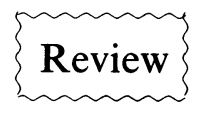

\title{
Recent Development in Oral Vaccine Delivery Systems
}

\author{
A. E. Ellis \\ SOAFD Marine Laboratory PO Box 101, Victoria Road \\ Aberdeen AB9 8DB Scotland, UK
}

(Received September 26, 1995)

\begin{abstract}
Oral delivery of vaccines is the least effective method of vaccinating fish. However, anal intubation of antigens is very effective in inducing high serum antibody levels and protection against vibriosis and enteric redmouth. It is generally considered that degradation of antigen in the stomach and anterior intestine prevents immunostimulation taking place and that if antigens could be protected during passage through the foregut effective immunization could be achieved.

Recent attempts to protect antigens have included the concomitant administration of antacids and antiproteases. Protective coating procedures have included encapsulation of particulated antigen with methacrylic acrylic acid polymers which resist acid but dissolve in the high $\mathrm{pH}$ of the intestine.

Incorporation of antigen into microparticles of poly lactide co-glycolide polymers protects against enzyme degradation and allows increased uptake of intact antigen.

Certain substances can enhance uptake of antigen by the intestine of fish and have adjuvant activity, such as cholera toxin $\beta$-subunit and Quil-A saponin.

Overcoming problems of palatability of oral vaccines delivered to fish fry has been achieved by incorporating vaccine into live food such as Artemia. However, delivery of antigen to very young fish, before they are fully immunocompetent can induce immune suppression.

Many of these methods have improved the level of antigen uptake by the intestine and the antibody response in fish and the prospects for improving the efficacy of oral vaccination appear optimistic.
\end{abstract}

Key words: vaccine, oral delivery, anal delivery, antacid, antiprotease, encapsulation, ECAMS, adjuvant

\section{Introduction}

Over the past decade the vaccination of Atlantic salmon smolts has become an integral part of the aquaculture industry and in Norway, Scotland and Ireland, virtually every individual fish is vaccinated at some time before transfer to sea water. Some 10 years ago the main vaccines used in salmon culture were against vibriosis and enteric redmouth (ERM). These vaccines work extremely well when administered by immersion. Just a decade ago (Egidius $e t$ al., 1986), a new pathogen was recognised as causing important economic losses in Norwegian salmon farms, namely Vibrio salmonicida, the causative agent of cold water vibriosis. A vaccine against this disease was developed quickly but bath administra- tion induced limited protection and injection delivery was promoted.

In Scotland, the major cause of loss in farmed salmon was from furunculosis, caused by the bacterium Aeromonas salmonicida, which also began to spread through Norwegian farms in the late 1980s. The widespread antibiotic resistance of this organism made the development of a vaccine imperative. A great deal of research indicated that immersion vaccination against furunculosis was ineffective, but injection of the vaccine in an oil adjuvant was highly effective. Under the enormous economic pressure from losses due to furunculosis, the industry quickly adopted an injection delivery programme with marked success. Over the last five years, injection vaccination of Atlantic salmon smolts has become routine and many commercial vaccines, especially in 
Table 1. Protection against vibriosis in salmonids immunised by various routes

\begin{tabular}{lccc}
\hline \hline \multirow{2}{*}{ Vaccine preparation and route } & \multicolumn{3}{c}{ \% mortality } \\
\cline { 2 - 4 } & Lillehaug (1984) & Amend and Johnson (1981) & Johnson and Amend (1983) \\
\hline Control & 65 & 52 & 55 \\
Intraperitoneal injection & 0 & 0 & - \\
Immersion & 6 & 4 & 0 \\
Anal (intubation) & 16 & - & - \\
Oral (intubation) & - & 27 & - \\
Oral (in feed, for 15 days) & 29 & - & - \\
Oral "prills" (intubation) & 44 & - &
\end{tabular}

* not tested.

Norway, are tri- or quadrivalent, i.e. contain a mixture of vaccines comprised of the classical Vibrios, $Y$. ruckeri, $V$. salmonicida and $A$. salmonicida in an oil emulsion. While most farms perform the injection vaccinations manually, automated injection machines have become available.

Injection vaccination has certain restrictions and costs. It is expensive in staff time, stressful to the fish and the practicalities impose a minimum size limit on the fish to be vactinated which is about $15 \mathrm{~g}$. Oil adjuvants also induce undesirable side effects such as visceral granulomas which may reduce carcass value. Thus, while injection is acceptable for Atlantic salmon smolts, the more convenient methods of immersion and oral administration are desirable and for other fish species where vaccination is required when they are smaller than $15 \mathrm{~g}$ these methods are essential.

For obvious reasons, oral delivery of vaccines is the most convenient but much experience with feeding vaccines simply mixed with feed has indicated that insufficient levels of protection are achieved by this method.

Oral delivery of ERM bacterins required in-feed delivery for at least several weeks and protection was of short duration (Ross and Klontz, 1965; Anderson and Ross, 1972). In the late 1970s it became clear that immersion delivery for ERM and $V$. anguillarum bacterins was superior to oral delivery in that it was more economical in vaccine dosage and gave higher and longer lasting protection (Amend and Johnson, 1981).

The reason why immersion was more effective than oral delivery was considered to be because the antigens were not absorbed as well through the gut compared with skin/gills and/or that the antigens were digested in the gut. Evidence is quite strong for the protective antigens in both the ERM and Vibrio bacterins being lipopolysaccharide (LPS) (Kawai and Kusuda, 1983) and it is possible that this antigen has certain properties facilitating uptake by skin/ gills. Nevertheless, the harsh digestive conditions in the anterior gut as a restricting factor for the efficacy of oral delivery was indicated by Johnson and Amend (1983) (Table 1) when they compared oral and anal delivery of Vibrio and ERM bacterins. Anal intubation provided an excellent level of protection which was equal to or better than the immersion method. The authors suggested that a means of protecting vaccines from inactivation by gastric fluids in the anterior intestine could lead to the development of effective oral delivery of vaccines to fish.

Since then a considerable amount of work has been done to improve antigen uptake by the gut of fish and to understand the mechanism of antigen absorption by the gut.

\section{The Potential of Intestinal Antigen Stimulation-Oral Versus Anal Delivery}

Rombout et al. (1986) demonstrated the remarkable potential for vaccines delivered to the hind intestine to stimulate high serum antibodies. Carp were immunised with a $\boldsymbol{V}$. anguillarum bacterin by i.m. injection or by anal intubation $\left(10^{9}\right.$ bacterial fish). The serum antibody responses of these fish were very similar, peaking at a titre of about 256 . However, in fish immunised by the oral route with the bacterin mixed with the feed and administered once or daily for 32 days, the serum antibody levels were barely detectable. 
These antibody response data to $V$. anguillarum are corroborated by protection studies. Johnson and Amend (1983) found that anal intubation with $V$. anguillarum or Yersinia ruckeri bacterins gave a better protection than oral intubation. The level of protection was even greater than that after immersion and could be compared with that achieved by injection (Amend and Jonson, 1981). The principle of protecting antigens from degradation in the anterior intestine and methods of enhancing antigen uptake from the gastro-intestinal tract has been examined in a variety of ways. While much work is still to be done before oral delivery of vaccines to fish become economically feasible, the future prospects continue to look optimistic.

\section{Neutralisation of Gastric Secretions}

\section{Antacids}

In an attempt to improve intestinal uptake of intact proteins by neutralising gastric $\mathrm{pH}$ and removing the mucus lining of the gut epithelium, McLean et al. (1990) orally intubated coho salmon with recombinant bovine somatotropin, alone or mixed with $\mathrm{NaHCO}_{3}$ and a detergent. The hormone was intubated once per week for seven weeks at a dose of $12.5 \mu \mathrm{g} / \mathrm{g}$ body weight. At the end of the treatment period the body weight was compared with that of untreated controls and with fish injected i.p. with 2.5 $\mu \mathrm{g}$ somatotropin/g body weight/week. The injected group had a significantly increased body weight compared with controls (saline intubated). While the group intubated with somatotropin alone showed a growth enhancement, the group intubated with the hormone in the presence of $\mathrm{NaHCO}_{3}$ and detergent grew significantly faster than controls and matched that of the injected fish.

An important aspect of this study, though not in itself concerned with vaccines, is that the absorbance of intact protein molecules, retaining their biological activity, does occur in fish and the level of absorbance can be enhanced by neutralising gastric $\mathrm{pH}$.

\section{Antiproteases}

The potential of soybean trypsin inhibitor (SBTI) to inhibit proteolytic degradation of proteins and increase intestinal uptake of intact protein was studied by McLean and Ash (1990). Horse-radish peroxidase (HRP) was orally intubated in rainbow trout with or without SBTI. The tissue concentrations of
HRP were significantly increased when the enzyme was delivered with SBTI.

\section{Encapsulated Antigens}

A variety of methods of coating antigens to protect them from degradation in the anterior intestine and release them in the posterior intestine to mimic anal intubation delivery has been attempted.

\section{"Prills" and acid resistant films}

Lillehaug (1989) explored the use of "prills", which are basically pellets $(8-10 \mathrm{mg}$ in weight $)$ comprising lyophilised vaccine incorporated into a matrix of saturated long chain fatty acids, or granulated lyophilised vaccine coated with an acid-resistant acrylic film. Both preparations were designed to protect a $V$. anguillarum bacterin during passage through the anterior intestine and dissolve in the hind-intestine to release the vaccine. The preparations were delivered by oral intubation and the level of protection against a vibriosis challenge was compared with fish orally intubated with unprotected vaccine or immunised by immersion or injection.

Table 1 shows the percentage mortality of the different groups of fish alongside data from similar experiments reported by Amend and Johnson (1981) and Johnson and Amend (1983) involving delivery of Vibrio vaccine by various routes for comparison.

Although the results of Johnson and Amend (1983) comparing oral and anal deliveries indicated that protection of the vaccine against gastric digestion might improve efficiency of oral delivery, this was not achieved by the preparations tested by Lillehaug (1989). Indeed, the opposite effect was observed. Possibly the vaccine was not only protected from degradation but may also have been protected from being absorbed by the encapsulation procedures.

While prolonged feeding and relatively high doses of vaccine have been used in most studies on administering vaccines in the feed (Fryer et al., 1978; Kawai and Kusuda, 1985; Amend and Johnson, 1981), the degree of protection achieved by a single intubation of a dose comparable to injection as reported by Lillehaug (1989) and Johnson and Amend (1983) (see Table 1) was relatively high.

Many studies (see below) have used oral intubation as an experimental method for investigating oral delivery of antigens and this, compared with mixing 
the antigen with the feed may be a crucial factor for efficacy. Intubating the antigen may allow it to pass through the stomach relatively quickly, reducing the possible influence of gastric digestion, and reach the intestine in a more concentrated form to stimulate a greater immune response. Lillehaug (1989) suggested that efforts to develop a method in which a vaccine is given orally in a more concentrated form as a "vaccine pellet" may be more successful than encapsulating the vaccine to prevent digestion, at least when lipopolysaccharides (LPS) are the main antigens of importance as is the case with $V$. anguillarum vaccines (Kawai and Kusuda, 1983).

\section{Enteric coated antigen microspheres (ECAMS)}

A similar approach to protecting antigens against gastric digestion was studied by Piganelli et al. (1994). However, in this case, and developing upon the work of Lillehaug (1989), the protected antigen was delivered in the feed over a prolonged period (30 days) and an LPS antigen was compared with a protein antigen, the latter being more sensitive to denaturation in the gut.

ECAMS were prepared by soaking dextrose beads in aqueous solutions of antigen and then coating with a film of acrylic polymer. This created an acid resistant film which dissolved in the high $\mathrm{pH}$ of the intestine. The antigens chosen were trinitrophenylated-LPS (TNP-LPS) and TNP-keyhole limpet haemocyanin (TNP-KLH)

TNP-LPS was administered to coho salmon in ECAMS mixed with feed for 30 days at three different doses $(10 \mu \mathrm{g}, 10 \mathrm{ng}$ and $1 \mathrm{pg}$ per day). Other groups of fish were immunised by i.p. injection or immersion with $10 \mu \mathrm{g}, 10 \mathrm{ng}$ or $1 \mathrm{pg}$ administered once. The serum antibody response in the immersion immunised fish was negligible. In the injected group, a dose-related antibody response was observed. In the case of the ECAM-groups, only those administered the high dose responded, but interestingly the peak titre was equal to that in fish immunised with the high dose delivered by injection.

The TNP-KLH was administered in ECAMS mixed with feed at doses of $100 \mu \mathrm{g}, 5 \mu \mathrm{g}$ and $0.5 \mu \mathrm{g}$ daily, for 30 days. Other groups of fish were immunised by anal intubation of TNP-KLH in saline (100 $\mu \mathrm{g}$, once) or by i.p. injection in saline $(100 \mu \mathrm{g}$, once). Of the ECAM-groups, only the low dose induced a serum antibody response and the peak titre was equal to that induced by injection immunisation, though it appeared later. Anal immunisation also induced peak serum antibody levels that were as high as i.p. immunisation.

These data once again demonstrate that anal immunisation can achieve a high serum antibody response and that the ECAMS system, given optimisation of the dose regime, is also capable of eliciting high serum responses by oral delivery of antigen mixed with the feed.

\section{Poly lactide co-glycolide (PLG) microparticles}

PLG is a biocompatible and biodegradable polymer and has been used in a wide variety of circumstances including pulsed delivery of pharmaceuticals in human medicine. Recently it has been used to entrap antigens within microparticles (less than 10 $\mu \mathrm{m}$ in diameter) and found to induce high serum antibody titres in mammals when either injected (Eldridge et al., 1991) or when administered orally (Challacombe et al., 1992). To date, little has been published on the application of PLG to immunisation of fish but current work suggests that intestinal uptake can be enhanced following oral intubation of antigen in PLG microspheres. Lavelle (1994) found that when human gamma-globulin (HGG) was orally intubated into rainbow trout, this antigen could be detected in the serum for up to six hours. However, using Western blotting analysis, only fragments of HGG were detected, indicating the antigen had been degraded in the intestine. On the other hand, HGG encapsulated in PLG could be detected in the serum for at least $24 \mathrm{~h}$ and it was estimated that over $60 \%$ of the antigen was still intact. The protection against enzymic degradation of antigens encapsulated in PLG, the ability of microparticles to be absorbed by the intestine and the timed release of the antigen (achieved by using PLG of differing physical properties which determine the rate at which the matrix of the polymer hydrolyses and disintegrates), offer exciting prospects for the delivery of oral vaccines in this medium.

\section{Oral Adjuvants}

\section{Cholera toxin $\beta$-subunit, $\mathrm{Al}(\mathrm{OH})_{3}$ and $\mathrm{NH}_{4} \mathrm{Cl}$}

Most adjuvants are designed for injection but some have been demonstrated, in mammals, to enhance antigen uptake from the intestinal tract. Jenkins et al. (1994a) investigated antigen uptake and serum antibody responses when tilapia were im- 
munised with HGG by injection, anal intubation or oral intubation. For all routes the HGG was administered in saline or mixed with cholera toxin $\beta$-subunit (CTB) aluminium hydroxide, or ammonium chloride.

The orally intubated fish were analysed for uptake of HGG into the plasma. Compared with HGG intubated in saline, only the CTB enhanced the plasma concentration of the antigen.

Serum antibody titres of all groups were monitored for $\mathbf{3 5}$ days following immunisation. Injection (i.p.) induced significantly higher titers than any other method, though none of the potential adjuvants enhanced the antibody response compared with antigen injected in saline. The antibody responses induced by oral and anal intubation were similar to each other. The peak titres induced by antigen administered with $\mathrm{CBT}$ and $\mathrm{Al}(\mathrm{OH})_{3}$ were enhanced compared with antigen in saline while $\mathrm{NH}_{4} \mathrm{Cl}$ had no effect compared with the latter.

It is noteworthy that in tilapia, the anal administration of the protein antigen, HGG, did not induce an antibody response that was enhanced compared with oral administration and these enteric routes induced significantly lower antibody titres as compared with injection immunisation. This contrasts with data reported by Rombout et al. (1986) and Piganelli et al. (1994) who immunised carp with ferritin and coho salmon with TNP-KLH (referred to above), respectively, and who reported that anal intubation induced equally high serum antibody titres as injection. This suggests that the superior antibody responses obtained from anal as compared with oral intubation may depend upon the species of fish and/or the antigen used.

\section{Quil-A saponin}

Quil-A saponin is a well known adjuvant in mammals and has been found to enhance immune responses when given with antigen by injection or orally. Jenkins et al. (1994b) investigated the effect on the antibody response of tilapia to HGG when this antigen was administered by i.p. injection or oral intubation with and without Quil-A saponin. Both systemic (serum) and local (bile and skin mucus) antibody responses were monitored. Injection induced the highest titres in all three samples. Oral intubation of HGG alone induced very low antibody titres while the presence of Quil-A significantly enhanced the titres in serum, bile and skin mucus.

\section{Oral Delivery to Fish Larvae}

Many fish species used in aquaculture have very small larval stages which require the feeding of live planktonic prey. The concept of using such live diets to deliver vaccines was investigated by Kawai et al. (1989). These workers incubated the rotifier Brachionus plicantilis with a Vibrio bacterin and then fed them to juvenile ayu for 8-14 days. Significant protection was achieved against vibriosis.

Campbell et al. (1993) investigated the potential of using Artemia (brine shrimp) nauplii as a vehicle for delivering $V$. anguillarum bacterins. The uptake of the bacterin by the nauplii was determined but the authors expressed some doubt as to whether it would be sufficient to induce immune protection in recipient fish.

\section{Oral Boosting or Priming}

Given the background information that oral delivery of vaccines had limited efficacy, some workers have investigated the potential of oral delivery to enhance the antibody response when given prior to or subsequent to injection vaccination and thereby improve efficacy of the latter.

Vinitnantharat and Plumb (1993) monitored the antibody response of channel catfish vaccinated by injection with an antigen extract of the bacterium Edwardsiella tarda and then fed antigen-impregnated feed every 5 th or 10th day beginning 39 days after vaccinating by injection. Antibody titres in the control injected fish, not receiving the oral booster, peaked at day 20 and declined continuously thereafter. However, antibody titres in the orally boosted fish increased again.

The effect of oral priming juvenile carp and seabream (Sparus aurata) with a $V$. anguillarum vaccine incorporated into Artemia nauplii was investigated by Joosten et al. (1995). The bio-encapsulated antigen was fed to 58 day old carp and seabream for $2.5 \mathrm{~h}$. Ten weeks later the fish received an i.m. injection of the bacterin and three weeks after this the fish were bled and assayed for serum anti-Vibrio antibody titres. These fish had significantly higher antibody levels compared with the control groups which did not receive the oral priming.

A further aspect of this work, with obvious importance, studied oral priming in carp younger than 58 
days, namely 15 and 29 days old. An opposite effect of oral priming in these fish was observed in that the antibody titres following i.m. injection were significantly lower than the control groups not receiving the oral priming. This indicates that very young carp can develop immunological tolerance to intestinal exposure to antigens which lasts for at least 10 weeks; an important feature in developing protocols for oral priming of juvenile fish.

\section{A Potential Problem for Oral Vaccination-Oral Hyposensitisation}

In mammals, antigens in the diet can stimulate a local response in the gut mucosa and at the same time suppress a systemic response to the antigen when subsequently administered parenterally. This phenomemon is known as "oral hyposensitisation" (Mowat, 1987). The mechanism is still not fully understood and is dependent upon a complex set of factors including nature of the antigen (it is more readily induced by soluble $\mathrm{T}$-dependent antigens), dosage, frequency of administration and age of the animal (Faria et al., 1993). Generally speaking, high doses of antigen fed for long periods can induce suppression of the systemic response while low doses fed for shorter periods can prime or even stimulate a serum antibody response.

Some evidence for "oral hyposensitisation" in fish has been reported by Davidson et al. (1994). Rainbow trout were i.p. injected with HGG. At the same time, the fish were administered $2 \mathrm{mg}$ HGG by oral or anal intubation. The intubation was repeated twice weekly for eight weeks. Treatment suppressed the serum antibody response compared with the control (saline intubated) group but the effect was dose dependent $(20 \mathrm{mg}$ and $0.2 \mathrm{mg}$ HGG administered orally on each occasion, did not affect the antibody response).

When fish were administered HGG by oral intubation for five or 10 days prior to i.p. injection, no effect of the oral exposure was observed on the antibody response. Furthermore, the suppressive effect of oral delivery subsequent to i.p. injection of HGG was not observed with an Aeromonas salmonicida bacterin. Indeed, the data indicated that the serum antibody response was enhanced to this antigen by this treatment.

While under certain circumstances of high dose oral intubation of protein antigen, oral hyposensitisa- tion may be inducible in fish, the current experience suggests that it should not interfere with regimes of administration being sought in aquaculture.

\section{Future Development in Oral Vaccination-Lectins and Live Vaccines}

As described above, many studies have demonstrated that, using anal intubation, the hind-intestine of fish is sensitive to antigen stimulation and an important site of antigen uptake (Rombout et al., 1989; Joosten et al., 1995) resulting in high serum antibody titres that can equal the response achieved by injection immunisation. Thus, the major hurdles to overcome for achieving effective oral delivery of vaccines appear to be protecting them against degradation and improving uptake. Certain encapsulation procedures such as ECAMS (Piganelli et al., 1994) show great promise in achieving this while procedures, e.g. prills (Lillehaug, 1989), have failed.

\section{Lectins}

PLG microspheres can protect antigens from enzymic degradation in the gut and are capable of being taken up by the intestinal mucosa in mammals (Eldridge et al., 1989). One approach to increasing this uptake is to incorporate lectins into the microspheres which make them very adhesive to the epithelial cells (Shahin et al., 1992; Santiago et al., 1995).

\section{Live vaccines}

The ability of attenuated Salmonella typhi to invade, persist and proliferate in the human gut associated lymphoid tissues and induce a protective immune response, without causing clinical symptoms, is well known (Curtiss et al., 1989). This observation has prompted many workers to introduce genes encoding antigens for other organisms into modified strains of Salmonella, using them as vectors for selective delivery of antigen via the intestine (Sadoff et al., 1988).

A genetically attenuated strain of $A$. salmonicida (AroA mutant) has been produced by Vaughan et al. (1993) which has been shown to be avirulent, persist in trout tissues for up to 12 days post injection and induce strong protective immunity. It is feasible that this mutant could be used for oral administration and furthermore, genes from other fish pathogens, especially viruses, could be cloned into and expressed 
by the AroA mutant thus acting as a vector for other vaccines.

\section{Conclusions}

There remains much research to be done to develop oral vaccines for fish but the potential of antigen delivery to the gut, as demonstrated by the high immune responses achieved by anal intubation, offers many advantages.

Oral delivery of vaccines can lead to higher antibody titres in skin mucus than are achieved with injection vaccination, e.g. carp immunised with a $V$. anguillarum bacterin (Rombout et al., 1989). If protection against pathogens is based upon antibody responses in the mucosal surfaces of fish, oral delivery may be more efficacious than injection. More information concerning the local immune responses in the gut, skin and gills, induced by oral immunisation is needed to assess these potentials.

Optimisation of doses for oral delivery and duration of administration is required. In order to stimulate high systemic immune responses, means of protecting the antigen from degradation in the anterior gut and enhancing uptake in the hind-intestine, especially when antigen is given in the feed, appears to be of paramount importance. Some of the encapsulation processes already attempted show definite promise but many modifications to these processes still remain to be tested.

\section{Acknowledgements}

This work was partly funded by the invitation program of Tokyo University of Fisheries and presented at Fall Session of the Meeting of the Japanese Society of Fish Pathology, Ise, Mie prefecture, September 25-26, 1995.

\section{Referecnes}

Amend, D. F. and K. A. Johnson (1981): Current status and future need of Vibrio anguillarum bacterins. Dev. Biol. Stand., 49, 403-417.

Anderson, D. P. and A. J. Ross (1972): Comparative study of Hagerman redmouth disease oral bacterins. Proc. Fish. Cult., 34, 226-228.

Campbell, R., A. Adams, M. F. Tatner, M. Chair and P. Sorgellos (1993): Uptake of Vibrio anguillarum vaccine by Artemia salina as a potential oral delivery system to fish fry. Fish Shellfish Immunol., 3, 451-459.

Challacombe, S. J., H. Rahman, H. Jeffery, S. S. Davis and D. T. O'Hagen (1992): Enhanced secretory IgA and systemic IgG antibody responses after oral immunisation with biodegradable microparticles containing antigens. Immunol., 76, 164-168.

Curtiss, R., S. M. Kelly, P. P. Gulig and K. Nakayama (1989): Selective delivery of antigens by recombinant vaccine. Curr. Top. Microbiol. Immunol., 146, 35.

Davidson, G. A., A. E. Ellis and C. J. Secombes (1994): A preliminary investigation into the phenomenon of oral tolerance in rainbow trout (Oncorhynchus mykiss, Walbaum, 1792). Fish Shellfish Immunol., 4, 141-151.

Egidius, E., R. Wiik, K. Anderson, K. A. Hoff and B. Hjeltnes (1986): Vibrio salmonicida sp. nov., a new fish pathogen. Int. J. syst. Bact., 36, 518-520.

Eldridge, J. H., J. K. Staas, J. A. Meulbroek, J. R. McGhee, T. R. Tice and R. M. Gilley (1991): Biodegradable microspheres as a vaccine delivery system. Mol. Immunol., 28, 287-294.

Faria, A. M., G. Garcia, M. J. C. Rios, C. L. Michalaros and N. M. Vaz (1993): Decrease in susceptibility to oral tolerance induction and occurrence of oral immunisation to ovalbumin in 20-38 week old mice. The effect of interval between oral exposures and the rate of antigen intake in the oral immunisation. Immunol., 78, 147-151.

Fryer, J. L., J. S. Rohovec and R. L. Garrison (1978): Immunisation of salmonids for control of vibriosis. Mar. Fish. Rev., 40, 20-23.

Jenkins, P. G., A. B. Wrathmell, J. E. Harris and A. L. Pulsford (1994a): The effect of different adjuvants on intestinal antigen absorption and subsequent immune responses of the tilapian Oreochromis mossambicus. Fish Shellfish Immunol., 4, 167-177.

Jenkins, P. G., A. B. Wrathmell, J. E. Harris and A. L. Pulsford (1994b): Systemic and mucosal immune response to enterically delivered antigen in Oreochromis mossambicus. Fish Shellfish Immunol., 4, 255-271.

Johnson, K. A. and D. F. Amend (1983): Efficacy of Vibrio anguillarum and Yersinia ruckeri bacterins applied by oral and anal intubation of salmonids. J. Fish Dis., 6, 473-476.

Joosten, Ph. H. M., M. Aviles-Trigueros, P. Sorgeloos and J. H. W. M. Rombout (1995): Oral vaccination of juvenile carp (Cyprinus carpio) and gilthead seabream (Sparus aurata) with bioencapsulated Vibrio anguillarum bacterin. Fish Shellfish Immunol., 5, 289-299.

Kawai, K. and R. Kusuda (1983): Efficacy of the lipopolysaccharide vaccine against vibrosis in cultured ayu. Bull. Jap. Soc. Sci. Fish., 49, 511-514.

Kawai, K. and R. Kusuda (1985): Field testing of oral Vibrio anguillarum bacterin in pond-cultured ayu. Fish Pathol., 20, 413-419.

Kawai, K., S. Yamamoto and R. Kusuda (1989): Plank- 
ton-mediated oral delivery of Vibrio anguillarum vaccine to juvenile ayu. Nippon Suisan Gakkaishi, 55, 35-40.

Lavelle, E. C. (1994): Gastrointestinal antigen processing and its relevance to enteric vaccine delivery in rainbow trout, Oncorhynchus mykiss (Walbaum, 1792). PhD Thesis, Plymouth University, Devon, UK.

Lillehaug, A. (1988): Oral immunisation of rainbow trout, Salmo gairdneri Richardson, against vibriosis with vaccines protected against digestive degradation. J. Fish Dis., 12, 579-584.

McLean, E. and R. Ash (1990): Modified uptake of the protein antigen horse radish peroxidase (HRP), following oral delivery to rainbow trout, Oncorhynchus mykiss. Aquacult., 87, 373-379.

McLean, E., E. M. Donaldson, H. M. Dye and L. M. Souza (1990): Growth acceleration of coho salmon (Oncorhynchus kisutch) following oral administration of recombinant bovine somatotropin. Aquacult., 91, 197-203.

Mowat, A. McI. (1987): The regulation of immune responses to dietary protein antigens. Immunol. Today, $\mathbf{8}$, 93-98.

Piganelli, J. D., J. A. Zhang, J. M. Christensen and S. L. Kaattari (1994): Enteric coated micropheres as an oral method for antigen delivery to salmonids. Fish Shellfish Immunol., 4, 179-188.

Ross, A. J. and G. H. Klontz (1965): Oral immunisation of rainbow trout (Salmo gairdneri) against an etiological agent of "redmouth" disease. J. Fish. Res. Bd Can., 22, 713-719.

Sadoff, J. C., W. R. Ballou, L. S. Baron, W. K. Majariau, R. N. Brey, W. T. Hockmeyer, J. F. Young, S. J. Cryz, G. H. Lowell and J. D. Chulay (1988): Oral Salmonella typhimurium: vaccine expressing circumsporozoite protein protects against malaria. Science, 240, 336-338.

Santiago, N., S. Haas and R. A. Baughman (1995): Vehicles for oral immunisation. In: Vaccine design: the subunit and adjuvant approach. (ed. by M. F. Powell, and M. J. Newman). Plenum Press, NY, pp. 413-438.

Shahin, R. D., D. F. Amsbaugh and M. F. Leef (1992): Mucosal immunisation with filamentous hemagglutinin protects against Bordetella pertussis respiratory infection. Infect. Immun., 60, 1482-1488.

Vaughan, L. M., P. R. Smith and T. J. Foster (1993): An aromatic-dependent mutant of the fish pathogen Aeromonas salmonicida is attenuated in fish and is effective as a live vaccine against the salmonid disease furunculosis. Infect. Immun., 61, 2172-2181.

Vinitnantharat, S. and J. A. Plumb (1993): Protection of channel catfish Ictalurus punctatus following natural exposure to Edwardsiella ictaluri and effects of feeding antigen on antibody titres. Dis. Aquat. Org., 15, 31-34. 